\title{
Surgical management of post BCG vaccine lymphadenitis, comparative retrospective study
}

\author{
Mohamed E Hassan, MD, FEBPS
}

Department of Pediatric Surgery, Zagazig University, Egypt.

Introduction: Adverse reactions following BCG vaccination are uncommon, with an estimated prevalence of 0.4 per 1000 vaccines. The options for surgical management of post $B C G$ vaccine suppurative lymphadenitis are incision and drainage, total excision, or simple needle aspiration. The aim of the study is to compare the outcome of surgical treatment of suppurative post BCG lymphadenitis using simple needle aspiration versus primary excision.

Patients and methods: A retrospective file review was done for all children who presented, with a diagnosis of post BCG vaccine suppurative lymphadenitis (axillary and/ or supraclavicular), to the pediatric surgery clinic in two tertiary pediatric surgery centers during the period from January 2007 to January 2013.

Results: The study population included 91 patients, 38 in Group 1 versus 53 in Group 2. There were 60 males and 31 females. Two groups were identified in the study, according to the type of management at the initial presentation, Group 1 who were managed with simple needle aspiration and Group 2 who were managed with primary surgical excision. Patients who received antituberculous treatment were excluded from the study.

Conclusion: Primary surgical excision for post BCG suppurative lymphadenitis showed better outcomes than needle aspiration in the current study population.

Key words: BCG, vaccine, lymphadenitis, children.

\section{Introduction:}

Bacillus Calmette-Guerín (BCG) is a live attenuated vaccine to prevent tuberculosis, routinely administered at birth as part of the World Health Organization global expanded immunization program. ${ }^{1}$

Adverse reactions following BCG vaccination are uncommon, with an estimated prevalence of 0.4 per 1000 vaccines. $^{2}$ Complications include erythema, blistering, abscess formation, regional lymphadenitis and keloid formation. ${ }^{3}$

The options for surgical management of post BCG vaccine suppurative lymphadenitis are incision and drainage, total excision, or simpleneedleaspiration. Thechoice of surgical management remains controversial.4-8

The aim of the study is to compare the outcome of surgical treatment of suppurative post BCG lymphadenitis using simple needle aspiration versus primary excision.

\section{Patients and methods:}

A retrospective file review was done for all children who presented, with a diagnosis of post BCG vaccine suppurative lymphadenitis (axillary and/ or supraclavicular), to the pediatric surgery clinic in two tertiary pediatric surgery centers during the period from January 2007 to January 2013.

Children with nonsuppurative lymphadenitis were excluded from the study.

Suppuration has been defined as "presence of fluctuation on palpation or pus on aspiration, the presence of a sinus, or large lymph node adherent to the skin with caseous lesions on excision". ${ }^{9}$

Two groups were identified in the study, according to the type of management at the initial presentation, Group 1 who were managed with simple needle aspiration and Group 2 who were managed with primary surgical excision. Patients who received 
antituberculous treatment were excluded from the study.

For Group 1, aspiration was done weekly for the first one month then monthly for 4 months (if during follow up no fluctuation were observed, no aspiration was done). The aspiration was done in the outpatient clinic under complete aseptic technique with a 19 Gauge needle and was sent for AFP and routine culture sensitivity test.

All patients in group 2 received a single dose of Amoxicillin/ Clavulonic acid before surgery.

Data collected included, patients demographics, site of suppurative lymphadenitis, the duration between BCG vaccination and suppurative lymphadentitis, number of aspirations, culture results of the pus in both groups, time to complete resolutions (calculated as the time frame from the first clinic visit till complete resolution of the suppurative lymphadenitis), the incidence of failed aspirations in group 1 and the incidence of wound infection in group 2 .

Data were tabulated and statistically analyzed using SPSS version 17, Chi square and $t$ tests were used to test for statistical significant difference between both groups. $\mathrm{P}<0.05$ was considered as statistically significant difference.

\section{Results:}

The study population included 91 patients, 38 in Group 1 versus 53 in Group 2. There were 60 males and 31 females Table (1).

There was statistically significant less time in group 2 for the complete resolution of the post BCG suppurative lymphadenitis,

\section{Table (2).}

There were 39\% ( 15 patients) in group 1 who failed to be treated with frequent aspiration who were subjected for complete excision of the suppurative lymphadenitis. Postoperative infection in group 2 was encountered in 19\% (10 patients), $\left(X^{2}=4.7\right.$, $\mathrm{P}<0.05$ ).

\section{Discussion:}

Bacillus Calmette-Guérin (BCG) vaccine has a good safety profile in immune competent children and considerable risks such as disseminated BCG disease in individuals with immune deficiencies. However, some complications, including local subcutaneous infection, regional suppurative lymphadenitis may occur after BCG vaccination in immune competent children. ${ }^{10}$

Lymphadenitis presenting within 2 months of vaccination and larger nodes $(+1 \mathrm{~cm})$ may be less likely to resolve spontaneously. ${ }^{6}$ The onset of suppuration may be variable with cases presenting from one week to 11 months following vaccination (de Souza et al., 1983). ${ }^{11}$ Mean age at presentation of the current study was 3.5 versus 4.3 months for group 1 and 2 respectively.

The current study populations didn't show any statistical significant differences in the patient's demographic data. The time required for development of post BCG suppurative lymphadenitis and the site of suppurative lymphadenitis was not different statistically between both groups.

$53 \%$ versus $60 \%$ in group 1 and 2 respectively, showed positive AFB cultures of the collected fluid. Bolger et al ${ }^{12}$ isolated $46 \%$ AFB in his study population from post BCG suppurative lymphadenitis.

None of our patients in the study were treated with antituberculuos medications. Two studies, one comparing isoniazid with erythromycin ${ }^{13}$ and the other a controlled placebo study, ${ }^{14}$ showed no clear benefit from the use of either of these 2 agents for the treatment of suppurative lymphadenopathy. There is no convincing evidence that medical interventions, including use of antituberculous agents, hasten recovery. ${ }^{15,16}$

The time required for complete treatment of the post BCG suppurative lymphadenitis was 135 versus 48 days in group 1 and 2 respectively, which was statistically significant.

$39 \%$ in group 1 failed to be treated with frequent aspiration, which is considered a complication of this type of surgical treatment. $19 \%$ in group 2 who developed postoperative wound infection were treated according to wound cultures. 7 versus 3 wounds showed gram positive and gram negative organisms 
Table 1: Demographics of both groups.

\begin{tabular}{|l|l|l|l|l|}
\hline \multicolumn{2}{|c|}{} & $\begin{array}{l}\text { Group 1 (38) } \\
\text { (Aspiration Group) }\end{array}$ & $\begin{array}{l}\text { Group 2 (53) } \\
\text { (Excision Group) }\end{array}$ & X2/t, P \\
\hline \multirow{2}{*}{ Sex } & Male & $26(68 \%)$ & $34(64 \%)$ & X2 $=0.18$ \\
\cline { 2 - 4 } & Female & $12(32 \%)$ & $19(36 \%)$ & P $>0.05$ \\
\hline $\begin{array}{l}\text { Mean Age at } \\
\text { Presentation }\end{array}$ & $3.5 \pm 1.9$ months & $4.3 \pm 2.4$ months & $\begin{array}{l}\mathrm{t}=1.7 \\
\mathrm{P}>0.05\end{array}$ \\
\hline
\end{tabular}

Table 2: Variables in both groups

\begin{tabular}{|c|c|c|c|c|}
\hline & $\begin{array}{l}\text { Group } 1 \text { (38) } \\
\text { (Aspiration Group) }\end{array}$ & $\begin{array}{l}\text { Group } 2 \text { (53) } \\
\text { ( Excision Group) }\end{array}$ & $\mathrm{X} 2 / \mathrm{t}, \mathrm{P}$ \\
\hline \multicolumn{2}{|c|}{$\begin{array}{l}\text { Mean duration between BCG vaccine and } \\
\text { suppurative lymphadentitis }\end{array}$} & $3.1 \pm 1.9$ Months & $4 \pm 2.5$ Months & $\begin{array}{l}t=1.9 \\
P>0.05\end{array}$ \\
\hline \multirow{3}{*}{$\begin{array}{l}\text { Site of BCG } \\
\text { Lymphadenitis }\end{array}$} & Supraclavicular & $5(13 \%)$ & $6(11 \%)$ & \multirow{3}{*}{$\begin{array}{l}X 2=0.7 \\
P>0.05\end{array}$} \\
\hline & Axillary & $30(79 \%)$ & $40(75 \%)$ & \\
\hline & Combined & $3(8 \%)$ & $7(13 \%)$ & \\
\hline \multicolumn{2}{|c|}{ AFB Positive Cultures of Aspirated Fluid } & $20(53 \%)$ & $32(60 \%)$ & $\begin{array}{l}\mathrm{X} 2=0.5 \\
\mathrm{P}>0.05\end{array}$ \\
\hline \multicolumn{2}{|c|}{ Mean Time to complete treatment } & 135 Days & 48 Days & $\begin{array}{l}t=97.7 \\
P<0.05\end{array}$ \\
\hline
\end{tabular}

respectively, which required local wound care and topical antibiotics. None of the infected wounds grew AFB cultures. Bolger et al isolated Staphylococcus aureus from five patients out of 25 (20\%) who required surgical excision of suppurative lymphadenitis. ${ }^{12}$

\section{Conclusion :}

Primary surgical excision of post BCG suppurative lymphadenitis was associated with statistically significant less time for complete surgical treatment than needle aspiration. Primary surgical excision was associated with less surgical complication rate than the aspiration group. Primary surgical excision for post BCG suppurative lymphadenitis showed better outcomes than needle aspiration in the current study population.

\section{Reference:}

1- Cuello-García CA, Pérez-Gaxiola G, Jiménez Gutiérrez C: Treating BCG-induced disease in children. Cochrane Database Syst Rev 2013; 31; 1.
2- Lotte A, Wasz-Hockert O, Poisson N, et al: Second IUATLD study on complications induced by intradermal BCG vaccination. Bull Int Union Tuber 1988; 63: 47-59.

3- Ghattaura A, Eley KA, Molenaar E, Smith G: A case of extensive ulcerating vasculitis following a BCG vaccination. $J$ Plast Reconstr Aesthet Surg 2009; 62(8): 286-289.

4- J Mark Fitz Gerald: Management of adverse reactions to Bacille Calmette-Guérin Vaccine. Clinical Infectious Diseases 2000; 31(Suppl 3): 75-76.

5- Bolger T, O’Connell M, Menon A, Butler K: Complications associated with the Bacille Calmette-Guérin vaccination in Ireland. Arch Dis Child 2006; 91: 594-597.

6- Caglayan S, Arikan A, Yaprak I, et al: Management of suppuration in regional lymph nodes secondary to BCG vaccination. Acta Paediatr Jpn 1991; 33: 699-702.

7- Sataynarayana S, Mathur AD, Verma Y, et al: Needle aspiration as a diagnostic tool and therapeutic modality in suppurative lymphadentitis following BCG vaccination. J Assoc Physicians India 2002; 50: 788-791.

8- Hengster P, Solder B, Fille M, et al: Surgical treatment of Bacille Calmette Guerin 
lymphadenitis. World J Surg 1997; 21: 520-523.

9- Lotte A, Wasz-Höckert O, Poisson N, Dumitrescu N, Verron M, Couvet E: BCG complications. Estimates of the risks among vaccinated subjects and statistical analysis of their main characteristics. Adv Tuberc Res 1984; 21: 107-193.

10- Kim DH, Choi CW: Chest wall abscess likely due to BCG vaccination in a child. Infection 2009; 37(3): 286-288.

11- De Souza GR, Sant'Anna CC, Lapa e Silva JR, Mano DB, Bethlem NM: Intradermal BCG vaccination complications-analysis of 51 cases. Tubercle 1983; 64(1): 23-27.

12- T Bolger, M O’Connell, A Menon, K Butler: Complications associated with the Bacille Calmette-Guérin vaccination in Ireland. Arch Dis Child 2006; 91: 594-597.
13- Hanley SP, Gumb J, MacFarlane JT: Comparison of erythromycin and isoniazid in treatment of adverse reactions to BCG vaccination. Br Med J [Clin Res] 1985; 290 : 970.

14- Cajlayan S, Yegin O, Kayran K, Timocin N, Kasiaga E, Gun M: Is medical therapy effective for regional lymphadenitis following BCG vaccination? Am J Dis Child 1987; 141: 1213-1214.

15- Noah P, Pande D, Johnson B, et al: Evaluation of oral erythromycin and local isoniazid instillation therapy in infants with Bacille Calmette Guerin lymphadenitis and abscesses. Pediatr Infect Dis J 1993; 12: 136-139.

16- Goraya J, Virdi V: Treatment of CalmetteGuerin bacillus adenitis: A metaanalysis. Pediatr Infect Dis J 2001; 20: 632-6334. 